www.jmscr.igmpublication.org

Impact Factor 5.84

Index Copernicus Value: 83.27

ISSN (e)-2347-176x ISSN (p) 2455-0450

crossref DOI: _https://dx.doi.org/10.18535/jmscr/v5i2.84

\title{
Recurrent Malignant Phyllodes Tumour of the Breast with Liposarcomatous Differentiation: A Rare Case Report and Review Of Literature
}

\section{Authors \\ Dr M Kumara Raghavendra Varma, Prof. Bhaskaran A, Dr Pavan B K, Dr Asadulla Baig}

\section{INTRODUCTION}

Phyllodes tumor of the breast usually is a biphasic fibroepithelial neoplasm. Malignant transformation is seen in 10 to $20 \%$ of cases and manifests in the form of fibrosarcomatous differentiation and rarely heterologous sarcomatous elements. Liposarcomatous differentiation is a rare entity among phyllodes tumors.

Spindle cell carcinoma is an unusual neoplasm commonly observed in the oral cavity ${ }^{1}$ and the laryn $\mathrm{x}^{2}$. However, on rare occasions it occurs in the breast $\mathrm{t}^{3,4}$. Primary pure breast sarcoma is a rare disease and constitutes $0.2-1.0 \%$ of all mammary malignancies ${ }^{5}$.

Primary pure breast sarcomas or advanced breast sarcomas are rare and mostly secondary to radiotherapy. Latter forms the majority of breast sarcomas. Reported 5-year survival rates for patients with breast sarcoma range from 40 to $91 \%$ 6,7,8

Sarcomatous stromal elements, including angiosarcoma, leiomyosarcoma, and rhabdomyosarcoma, are infrequent in malignant phyllodes tumors. Liposarcomas usually show stromal components of phyllodes tumors. Liposarcomatous differentiation is confirmed by noting well differentiated, myxoid, round cell, and pleomorphic liposarcomatous elements. The finding of a malignant heterologous element places the tumor into a malignant category.

\section{CASE REPORT}

A 48-year-old woman was admitted to our hospital with a 1-month history of recurrence of right breast lump with rapid progression following mastectomy 1 year back for previous benign phyllodes without any known personal or family history of malignancies. Lump associated with serous discharge from nipple.

Physical examination revealed a lump of $17 * 9$ $\mathrm{cm}$ in diameter in the right breast with satellite lesions around nipple areola complex, fixed to underlying muscle. Excisional biopsy was performed, and histopathological examination revealed recurrent phyllodes tumor. The patient underwent modified radical mastectomy and pathological examination of the specimen revealed malignant spindle cell lesion of right breast - Liposarcomatous differentiation of recurrent phyllodes.

Patient was followed up with metastatic workup and started on chemo - radiation postoperatively and is undergoing routine follow up visits. 


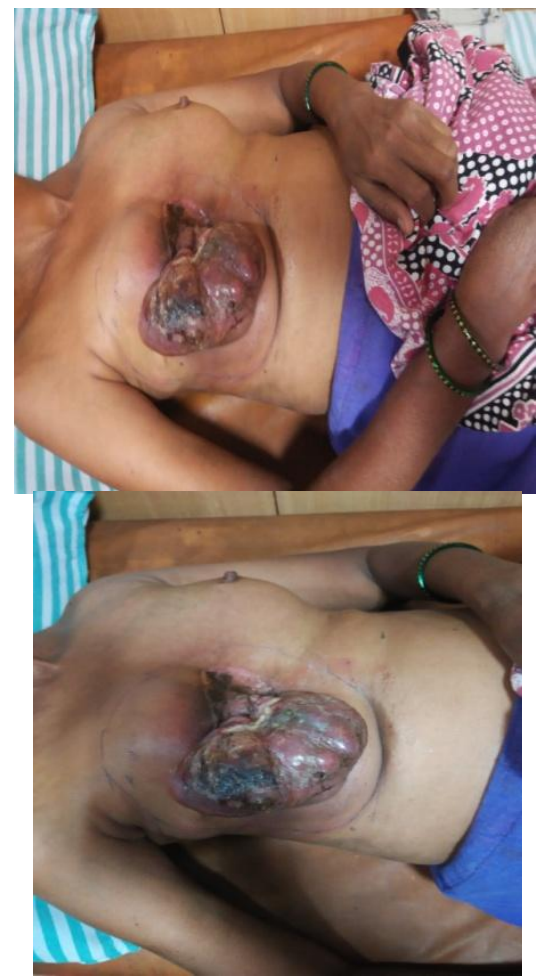

Figure 1: Picture showing pre-operative picture of recurrent phylloides tumor

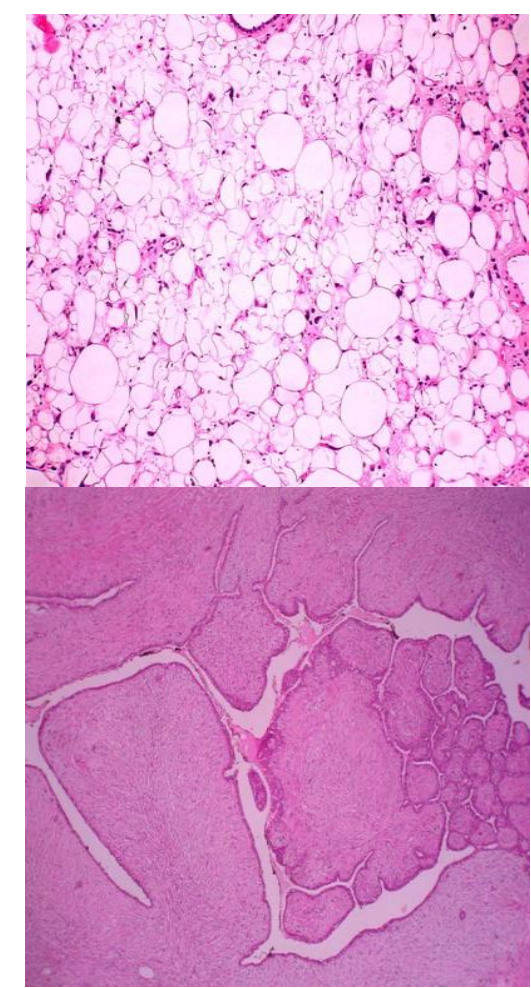

Figure 2: Histopathological slide showing Liposarcomatous differentiation and leaf like projections of Phylloides tumor

\section{DISCUSSION}

Sarcomas can occur anywhere in the body. $43 \%$ of sarcomas occur in the extremities with two-thirds of the extremity lesions occurring in the lower limb. $34 \%$ of sarcomas are intraabdominal, consisting of visceral (19\%) and retroperitoneal $(15 \%)$ lesions ${ }^{[4]}$. Breast sarcomas are rarely seen. Its incidence has been reported as $0.2 \%{ }^{(3)}$ or less than $0.5 \%{ }^{(4)}$.

It has been synonymously described as squamous carcinoma with spindle metaplasia, pseudosarcoma, sarcomatoid carcinoma, and carcinosarcoma ${ }^{(5)}$ Those that are seen are primary pure breast sarcomas or advanced breast sarcomas secondary to radiotherapy. The former is a rare disease, while the latter forms the majority of cases of breast sarcoma. As in the case of sarcomas of other body parts, treatment of breast sarcoma depends on the histological type, degree of differentiation, tumor size, and advancement of disease.

Secondary breast sarcoma is usually diagnosed at an advanced stage; therefore treatment is generally not successful. In local disease such as primary early breast sarcoma complete microscopic resection of the primary tumor is an important factor for local disease control, overall survival (OS), and Disease free survival (DFS).

Spindle cell carcinoma is a rare breast tumor. Its incidence has been reported as $0.2 \%$ (3) or less than $0.5 \%{ }^{(4)}$. It has been synonymously described as squamous carcinoma with spindle metaplasia, pseudosarcoma, sarcomatoid carcinoma, and carcinosarcoma ${ }^{(5)}$. However, immunohistochemical studies have recently suggested that spindle cell components are likely to be derived not from stromal but from epithelial cells. During the transformation from the epithelial shape to the spindle shape, mammary epithelial cells might acquire mesenchymal features and loose constitutive characteristics such as ER. Indeed, as presented here, spindle cell carcinoma of the breast has been found to be negative for $\operatorname{ER}^{(5,8)}$. Axillary dissection is generally thought to be unnecessary for most soft tissue sarcomas, since these tumors rarely metastasize via the lymphatics. Sarcoma secondary to radiotherapy is a well known complication of radiotherapy 
applied for breast carcinoma, and is associated with a poor prognosis. The standard and effective treatment for secondary breast sarcoma is surgery, but most cases are diagnosed at an advanced stage which might explain the poor prognosis. Although primary early breast sarcomas are treated surgically, survival rates are better compared to secondary breast sarcomas.

Other therapeutic options such as radiotherapy or chemotherapy will be necessary in these patients. Immunohistochemistry (IHC) may be useful to rule out other tumors such as non-mesenchymal malignant tumors or sarcomas with a specific line of differentiation. Desmin, vimentin, smooth muscle antigen, keratin, leukocyte common antigen, CD34, HMB45, EMA, and S-100 should all be analyzed in these sarcoma patients. However, some of the subtypes of primary breast sarcoma, such as fibrosarcoma, which have no specific immunological markers for this cell type may be indistinguishable.

Pathological prognostic factors play a considerable role in soft tissue sarcomas. Tumor grade is reported to be the single most important pathologic prognostic factor in soft tissue sarcomas.In primary breast sarcomas, adequate surgical excision of the tumor, grade, and tumor diameter seem to be the most important prognostic factors. In conclusion, most invasive breast neoplasms are epithelial tumors, and mesenchymal breast tumors are rarely seen and high degree of suspicion is required to detect these cases at an early stages which has prognostic significance especially.

\section{CONCLUSION}

In conclusion, most invasive breast neoplasms are epithelial tumors, and mesenchymal breast tumors are rarely seen and high degree of suspicion is required to detect these cases at an early stages which has prognostic significance especially.

\section{REFERENCES}

1. Someren A, Karcioglu Z, Clairmont A Jr. Polypoid spindle cell carcinoma (pleomorphic carcinoma). Oral Surg 1976;42:474-89

2. Randall G, Alonso W, Ogura J. Spindle cell carcinoma (pseudosarcoma) of the larynx. Arch Otolaryngol1975;101:63-6

3. Kaufman MW, Marti JR, Gallager HS, Hoehn JL. Carcinoma of the breast with pseudosarcomatous metaplasia. Cancer 1984;53:1908-17

4. Ellis IO, Bell J, Ronan JE, Elston CW, Blamey RW. Immunocytochemical investigation of intermediate filament proteins and epithelial membrane antigen in spindle cell tumours of the breast. $J$ Pathol1988;154:157-65

5. 5.Haberthur F, Feichter GE, Torhorst JK: Sarcoma of the breast: what is the contribution of immunohistochemistry and flow cytometry? [German] Geburtshilfe Frauenheilkd 1993;53:811-813

6. Pollard SG, Marks PV, Temple LN, Thompson HH:Breast sarcoma: aclinicopathologic review of 25 cases. Cancer 1990;66:941-944

7. 7.Callery CD, Rosen PP, Kinne DW: Sarcoma of the breast: study of 32 patients with reappraisal of classification and therapy. Ann Surg 1985;201:527-532

8. 8. Johnstone PA, Pierce LJ, Merino MJ, Yang JC, Epstein AH, DeLaney TF: Primary soft tissue sarcomas of the breast: local-regional control with postoperative radiotherapy. Int J Radiat Oncol Biol Phys 1993;27:671-675. 